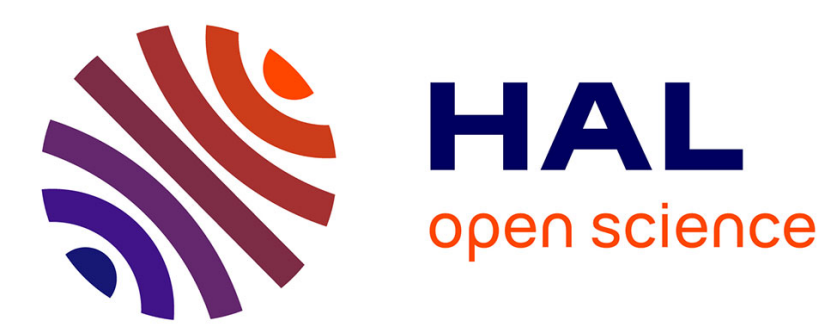

\title{
Analysis of Accelerating Detonation Using Large Activation Energy Asymptotics
}

R. Klein

\section{To cite this version:}

R. Klein. Analysis of Accelerating Detonation Using Large Activation Energy Asymptotics. Journal de Physique IV Proceedings, 1995, 05 (C4), pp.C4-443-C4-457. 10.1051/jp4:1995436 . jpa-00253741

\section{HAL Id: jpa-00253741 https://hal.science/jpa-00253741}

Submitted on 1 Jan 1995

HAL is a multi-disciplinary open access archive for the deposit and dissemination of scientific research documents, whether they are published or not. The documents may come from teaching and research institutions in France or abroad, or from public or private research centers.
L'archive ouverte pluridisciplinaire HAL, est destinée au dépôt et à la diffusion de documents scientifiques de niveau recherche, publiés ou non, émanant des établissements d'enseignement et de recherche français ou étrangers, des laboratoires publics ou privés. 


\title{
Analysis of Accelerating Detonation Using Large Activation Energy Asymptotics
}

\author{
R. Klein(1) \\ Institut für Technische Mechanik, RWTH Aachen, Templergraben 64, 52056 Aachen, Germany
}

\begin{abstract}
Galloping one-dimensional detonations exhibit a pulsating nonlinear instability characterized by rapid bursts of the detonation velocity followed by a slow-down and a longer re-acceleration. This has been observed experimentally as well as in numerical simulations, but to date there is no satisfactory theoretical description of this phenomenon that includes large amplitude deviations of the lead shock velocity. The present paper summarizes an extension of a recent analysis of the author based on large activation energy asymptotics. This earlier work exhibited a nonlinear slow-time instability for near-CJ detonations which leads to either an irreversible decomposition of the shock-reaction-zone complex or to a nonlinear continous acceleration, driving the wave speed away from the CJ-regime at an ever exceeding rate. Even though this analysis is based on an assumption of a slow characteristic evolution time scale, the insight gained seems to be worth a closer examination of the large amplitude regime that follows the above-mentioned acceleration stage. The present analysis, as well as the earlier study for the nearCJ regime, are in many respects close to the work by Buckmaster (1988), but they are not identical and seem to yield some new insight.
\end{abstract}

\section{INTRODUCTION}

One observes unsteady longitudinal oscillations experimentally as well as in one- and multidimensional direct numerical simulations based on Arrhenius one-step chemistry (Abuseif and Toong [1], Bourlioux and Majda [2]).

For highly overdriven fronts, an intriguing asymptotic theory, involving a modified Arrhenius reaction rate law, the limit of a specific heat ratio close to unity, low Mach number approximations in the shocked gas and small oscillation amplitudes of the detonation velocity has recently been presented by He and Clavin [3]. Here we are interested, in contrast, in order one overdrive and large amplitude excursions of the lead shock velocity.

The author has recently derived, via large activation energy asymptotics, a new inherently unsteady detonation front propagation law (Klein [4]). Let $d, \kappa$ denote the scaled detonation speed perturbation $\left(d=\theta\left(D^{\prime}-D_{\mathrm{CJ}}^{\prime}\right) / D_{\mathrm{CJ}}^{\prime}=O(1)\right)$ and the scaled lead shock curvature $\left(\kappa=\theta \ell_{I}^{\prime \mathrm{CJ}} \kappa^{\prime}\right)$, respectively. Here primes denote dimensional quantities, $D^{\prime}$ is the lead shock velocity of the detonation, $\ell_{I}^{\prime C J}$ the induction length of the associated CJ-wave, $\kappa^{\prime}$ the mean front curvature and $\theta=E_{a} / R T_{\mathrm{sh}}$ the nondimensional activation energy scaled with the post-shock state. Then the 
new front evolution equation reads

$$
\frac{\partial h(d, \kappa)}{\partial t}=g(d, \kappa)
$$

where $h(d, \kappa)=\ell_{I} / \ell_{I}^{\mathrm{CJ}}$ is the nondimensional induction length whose functional relation to $d_{3} \kappa$ is a result of the asymptotic analysis. In the steady limit the relation

$$
g(d, k)=0
$$

defines a detonation speed - curvature relation which has been derived recently independently by Yao and Stewart [5], He and Clavin [3] and Klein et al. [6]. Even though changes of the relevant dependent variables are assumed in this theory to occur on a time scale much longer than the induction time for a shocked particle, equation (1.1) is nevertheless an inherently unsteady front propagation law as it includes, in contrast to its steady limit version (1.2), the time derivatives of both the curvature and the detonation speed perturbation. For a discussion of the applicability of (1.1) and (1.2) as unsteady front evolution equations see Klein [4].

In the same paper the author addresses the stability problem for the steady limit solution in (1.2) based on the unsteady evolution equation (1.1). Even though this ansatz involves a neglection of all time scales comparable to the induction time, the result seems interesting:

For curved fronts, the upper branch of the $D$ - $\kappa$ - curve is time unstable, while the lower branch is temporally stable.

In the plane wave limit, the unsteady propagation law from (1.1) reveals a nonlinear long time instability, governed by the ODE

$$
\frac{d h}{d t}=\mathcal{F} \ln h, \quad \mathcal{F}=\text { const. }>0,
$$

for the time evolution of the induction length.

Klein [4] discusses the implications of the first of these statements regarding the behavior of curved fronts. Here we focus on the plane wave limit and observe that a wave which commences as a slightly perturbed CJ-detonation with $h(0)=1+\delta h_{0}$ must either collapse $\left(h \rightarrow 0\right.$ for $\delta h_{0}<0$ ) or fall apart $\left(h \rightarrow \infty\right.$ for $\left.\delta h_{0}>0\right)$ as time evolves. This collapse is associated with an ever increasing acceleration of the front and here we try to describe systematically what happens when the wave leaves the CJ regime.

Buckmaster [7] considers practically the same asymptotic regime discussed in the following. The principal idea of a collapsing shock-fire structure with an imbalance of energy release and shock dissipation leading to a rapid front acceleration on progressively shortening time scales has already been expressed clearly in his paper. Yet, in his analysis a secularity of one of the first order perturbation functions $\left(T^{1}\right)$ appears, which can be avoided. The consequence is a different detonation speed evolution equation.

\section{FORMULATION AND EARLIER RESULTS}

We consider the system of one-dimensional reactive Euler equations

$$
\begin{aligned}
\rho_{t}+(\rho \tilde{u})_{x} & =0 \\
(\rho \tilde{u})_{t}+\left(\rho \tilde{u}^{2}+p\right)_{x} & =0 \\
(\rho e)_{t}+(\tilde{u}[\rho e+p])_{x} & =0
\end{aligned}
$$


for mass, momentum and total energy, supplemented by one reaction progress equation

$$
(\rho \lambda)_{t}+(\rho \tilde{u} \lambda)_{x}=\rho w=\frac{1}{\theta} B(1-\lambda) \exp \left(\frac{\theta}{T}\right),
$$

in case of a single-step reaction model. Here $\rho, \tilde{u}, e, \lambda$ and $\theta$ are the density, velocity in a laboratory frame, total specific energy, the burnt gas mass fraction and the non-dimensional activation energy, respectively. The source term $\rho w$ represents the chemical species conversion.

Equations (2.1), (2.2) are closed with respect ot the pressure $p$ by specifying an equation of state. Here we chose the ideal gas equation,

$$
\rho e=\frac{p}{\gamma-1}+\frac{1}{2} \rho \tilde{u}^{2}+Q \rho(1-\lambda),
$$

with constant values for the isentropic exponent $\gamma$ and the specific heat of reaction $Q$.

The system (2.1) - (2.3) is in nondimensional form and we have assumed the following reference quantities for the scaling:

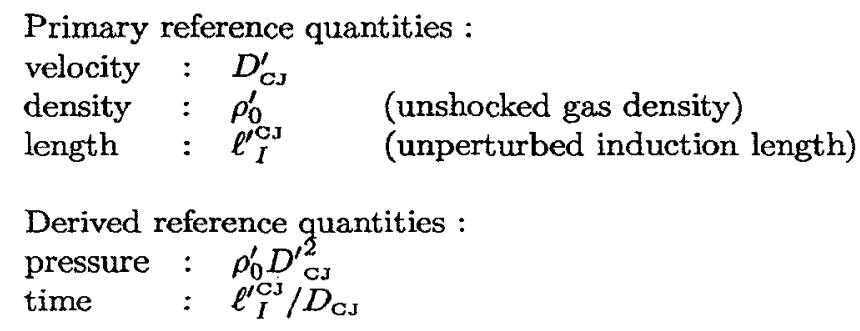

According to the Zel'dovič - von Neumann - Döring theory, we seek solutions that consist of a leading shock wave followed by a narrow zone of chemical activity. Thus, given the detonation speed $\mathrm{D}$, the solution has to satisfy the Rankine-Hugoniot jump conditions at $x=x_{\mathrm{sh}}(t)$, i.e., at the current location of the shock:

$$
\begin{aligned}
(\rho[\tilde{u}-D])_{\mathrm{sh}} & =-\rho_{0} D \\
\left(\rho[\tilde{u}-D]^{2}+p\right)^{+} & =\rho_{0} D^{2}+p_{0} \\
\left([\tilde{u}-D]\left[\rho e^{*}+p\right]\right)^{+} & =-D\left[\rho e^{*}+p\right]_{0}
\end{aligned}
$$

where $e^{*}$ is the total energy per unit mass in a shock-attached frame (replace $\tilde{\boldsymbol{u}}$ in (2.3) with $u=\tilde{u}-D$ !). Also, the lead shock is non-reactive, so that

$$
(\rho \lambda)_{\mathrm{sh}}=(\rho \lambda)_{0}=0 .
$$

In (2.4), (2.5), the superscript "s" denotes post-shock conditions, while the subscript "0" indicates conditions in the unshocked gas and the signs in (2.4) 1,3 indicate that the flow is from right to left in the front attached frame.

\subsection{The near-CJ regime}

In this section we summarize the analysis by Klein [4] for detonations that travel at close-to-CJ velocities. In the original analysis curvature effects were accounted for, but here we concentrate on plane waves in order to streamline the discussion. The underlying scaling assumptions for the following are: 
i) Deviations of the lead shock velocity from the Chapman-Jouguet speed are small and comparable to the inverse of the activation energy

$$
\frac{D-D_{\mathrm{CJ}}}{D_{\mathrm{CJ}}}=O\left(\frac{1}{\theta}\right) \quad \text { with } \quad \theta=\gg 1
$$

ii) The detonation structure is acoustically decoupled from the burnt gas flow so that, at the end of the reaction zone, the flow is sonic in the frame of reference attached to the "fire".

From Klein and Stewart [8], who presented a general set of front-attached coordinates suitable for the investigation of curved detonations, we extract the relevant system of governing equations here by specializing for one space dimension and keeping the terms indicating the time dependence of the detonation structure. The result is the following system of equations, which is equivalent to (2.1), but valid in the moving frame of reference:

$$
\begin{aligned}
\frac{\partial \rho}{\partial \tau}+\frac{\partial(\rho u)}{\partial n} & =0 \\
\frac{\partial}{\partial \tau}(u+D)+u \frac{\partial u}{\partial n}+\frac{1}{\rho} \frac{\partial p}{\partial n} & =0 \\
\frac{\partial E}{\partial \tau}+u \frac{\partial E}{\partial n}-\frac{p}{\rho^{2}}\left(\frac{\partial \rho}{\partial \tau}+u \frac{\partial \rho}{\partial n}\right) & =0 \\
\frac{\partial \rho \lambda}{\partial \tau}+\frac{\partial(\rho \lambda u)}{\partial n} & =\rho w
\end{aligned}
$$

Here $E$ is the the sum of thermal and chemical energy per unit mass of the gas mixture. The variables $n$ and $\tau$ denote a spatial coordinate measuring the distance from the lead shock and the time variable, respectively.

For the following it is convenient to introduce the "Master Equation" (Klein and Stewart [8]) that may be used to replace the energy equation in (2.7):

$$
\frac{1}{\rho} \frac{\partial p}{\partial \tau}-u \frac{\partial(u+D)}{\partial \tau}+\left(c^{2}-u^{2}\right) \frac{\partial u}{\partial n}-c^{2} \sigma w=0 .
$$

Here $c$ is the local speed of sound and the thermicity parameter $c^{2} \sigma$ is related to the chemical energy $Q$ via

$$
c^{2} \sigma=(\gamma-1) Q .
$$

\subsubsection{Induction zone analysis}

Following standard procedures in large activation energy asymptotics for ignition type problems we first consider the solution in the induction region that immediately follows the leading shock front and introduce the expansions

$$
D=D_{\mathrm{CJ}}\left(1+\frac{1}{\theta} d^{\star}(\tau)\right)
$$

for the lead shock velocity and

$$
\underline{u}=\underline{u}_{\mathrm{vN}}^{C J}+\frac{1}{\theta} \underline{u}^{(1)}(\xi, \tau)+O\left(\frac{1}{\theta^{2}}\right),
$$


where

$$
\underline{u}=(\rho, u, p, \lambda)
$$

is the solution vector and

$$
\xi=\theta n
$$

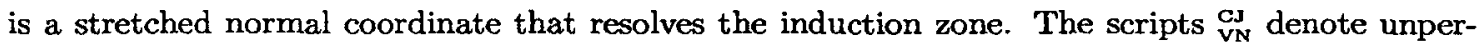
turbed post shock conditions.

Inserting this perturbation ansatz into the governing equations (2.7) $-(2.9)$ we first find that all the time derivatives are negligible to leading and first order. Thus the system reduces to the steady state flow equations. As the explicit time dependence of the solution is now purely parametrical, one may use the reaction progress equation

$$
\frac{\partial \lambda}{\partial \xi}=\frac{\hat{w}}{u}
$$

in order to replace the normal coordinate $\xi$. Notice that

$$
\hat{w}=w / \theta=O(1)
$$

throughout the reaction zone in the present asymptotic large activation energy regime. Then the Master equation (Klein, Stewart [8]) reads

$$
\frac{d u^{2}}{d \lambda}=\frac{2\left(c^{2} \sigma\right) u^{2}}{c^{2}-u^{2}}
$$

This is a closed form equation for either $c^{2}$ or $u^{2}$, provided one finds a functional dependence between $u^{2}, c^{2}$ and $\lambda$. For the quasi-onedimensional nozzle flow equations it is known that the total enthalpy is constant (see also Klein, Stewart [8]), i.e.,

$$
H=\frac{c^{2}}{\gamma-1}+\frac{u^{2}}{2}+Q(1-\lambda)=\text { const }=\frac{\mathrm{D}^{2}}{2}+\mathrm{Q}
$$

throughout the induction zone. Here we have used the fact that it is also constant across the lead shock and that $c^{2} \approx 0, u^{2}=D^{2}$ in the preshock state under the strong shock approximation. It is convenient (Klein et al. [6]) to solve for the perturbation $C$ of $c^{2}$ in the induction zone in terms of the stretched progress variable

$$
\Lambda=\theta \lambda
$$

One introduces

$$
c^{2}=c_{\mathrm{vN}}^{\mathrm{CJ}}\left(1+\frac{1}{\theta} C(\Lambda)\right)
$$

and finds the following ODE for $C(\Lambda)$ :

$$
\frac{d C}{d \Lambda}=\alpha
$$

where

$$
\alpha(\gamma)=\frac{3-\gamma}{4(\gamma-1)} .
$$

The asymptotic expansion of the lead shock jump conditions yields

$$
C(\Lambda=0)=2 d^{\star}
$$


and the exact solution for $C(\Lambda)$ reads

$$
C(\Lambda)=2 d^{\star}+\alpha \Lambda
$$

With $C(\Lambda)$ depending explicitly on the shock speed perturbation $d^{\star}$, we expect the induction length to vary nontrivially with time. The motion of the fire relative to the leading shock will introduce an inherently unsteady effect in the asymptotic solution of our problem and to determine its influence, we need to determine the induction length as a function of $d^{\star}$.

\subsubsection{The induction length:}

The induction length is the distance travelled by a mass element after it has been hit by the lead shock until it ignites and thus

$$
l_{I}=\left|\int_{t_{\mathrm{sh}}}^{t_{\mathrm{ign}}} u d t\right| .
$$

Using the expansion for the flow velocity $u$ in the shock-attached frame from (2.11) and noticing that along a particle path one has

$$
d t=\frac{d \lambda}{w}
$$

according to the reaction progress equation $(2.7)_{4}$, we rewrite $(2.24)$ as

$$
l_{I}=\left|u_{\mathrm{vN}}^{\mathrm{CJ}}\right| \int_{0}^{\infty} \frac{d \Lambda}{\theta B(\exp (-\theta) \exp (C(\Lambda))} .
$$

Inserting the exact solution for $C(\Lambda)$ from (2.23) one obtains

$$
h\left(d^{\star}\right)=\frac{l_{I}}{l_{I}^{\mathrm{C}}}=e^{-2 d^{\star}},
$$

where the induction length for the undisturbed wave

$$
l_{I}^{\mathrm{CJ}}=\frac{u_{\mathrm{VN}}^{\mathrm{CJ}}}{\alpha \theta B \exp (-\theta)}
$$

has been introduced.

Notice that the induction length depends on the detonation speed perturbation $d^{\star}$, which is varying nontrivially on the time scale considered. As a consequence, the induction length is changing too. In fact the order of magnitude of the velocity of the fire relative to the lead shock can be assessed to be

$$
i_{I}=l_{I}^{\mathrm{CJ}} \frac{\partial h}{\partial d^{\star}} \dot{d}^{\star}=O\left(\frac{1}{\theta} D_{\mathrm{cJ}}\right) .
$$

The estimate relies on the fact that we assume the time scale for changes of $d^{\star}$ to be given by $\theta l_{I} / D_{\mathrm{CJ}}$. Thus we find that the motion of the fire in the shock frame corresponds to a first order velocity in terms of $1 / \theta$ and we conclude that this motion will affect the asymptotic solution for the fire.

The induction zone solution exhibits a runaway as $\Lambda \rightarrow \infty$, since then $C(\Lambda)$ grows unboundedly and hence the perturbation expansion about the constant Chapman-Jouguet-von Neumann state becomes invalid. In the next subsection we will analyse the subsequent main reaction layer that is located in the immediate vicinity of the time dependent end of the induction zone. We will find that this main reaction region is quasisteady and that its structure is that of a weak deflagration. Assuming an acoustic decoupling of the reaction zone flow from the burnt gases, we impose sonic outflow from the fire to uniquely determine the solution. 


\subsubsection{The main reaction layer (fire)}

In the main reaction layer the reaction rate becomes exponentially large in comparison with its magnitude in the induction zone. It is thus even more justified to seek a steady-state - or quasisteady - solution in a suitable reference frame. The onset of the rapid heat release occurs at the end of the induction zone, so that we may seek a quasisteady solution of the form.

$$
\underline{u}=\underline{u}^{(0)}\left(\theta\left(n+l_{I}\right)\right)+\frac{1}{\theta} \underline{u}^{(1)}\left(\theta\left(n+l_{I}\right)\right)+o\left(\frac{1}{\theta}\right)
$$

with $\underline{u}$ as defined in (2.21). We expect the leading order solution not to depend on time, since the leading order induction zone solution is constant as well. The main reaction layer solution then satisfies the structure equation

$$
\frac{d \tilde{u}^{2}}{d \lambda}=\frac{\left(c^{2} \sigma\right) \tilde{u}^{2}}{c^{2}-\tilde{u}^{2}}
$$

where $\tilde{u}$ is the flow velocity in the fire frame. This is the master equation from (2.15) except for this change of the frame of reference. The exact leading order solution reads (Klein \& Stewart [8]):

$$
\tilde{u}^{(0)}(\ell)=-\frac{\gamma-\ell}{\gamma+1} D_{\mathrm{CJ}} \quad \text { where } \quad \ell=\sqrt{1-\lambda}
$$

and one has

$$
c^{2(0)}(\ell)=\frac{\gamma(1+\ell)(\gamma-\ell)}{(\gamma+1)^{2}} D_{\mathrm{CJ}}^{2}
$$

At first order one finds the perturbation equation for the velocity

$$
\frac{d u^{(1)}}{d \ell}=-2 \ell \frac{c^{2} \sigma}{\left(c^{2}-u^{2}\right)^{(0)}} u^{(1)}+\frac{2 \ell}{D_{\mathrm{CJ}}} \frac{\left(c^{2} \sigma\right) u^{2(0)}}{\left(c^{2}-u^{2}\right)^{(0)}}\left(c^{2}-u^{2}\right)^{(1)} .
$$

Now we seek the correct relation between $\left(c^{2}\right)^{(1)}$ and $\left(u^{2}\right)^{(1)}$, which should follow from the conservation of total enthalpy in the fire-frame: The total enthalpy (with respect to the fire frame) is defined by

$$
\tilde{H}=\frac{c^{2}}{\gamma-1}+\frac{\tilde{u}^{2}}{2}+Q(1-\lambda)
$$

and we have

$$
\tilde{u}=u+i_{I} \text {. }
$$

Thus up to, but excluding, the second order in $1 / \theta$ we have

$$
\tilde{H}=\left[\frac{c^{2}}{\gamma-1}+\frac{u^{2}}{2}+Q(1-\lambda)\right]+\frac{1}{\theta}\left(u_{\mathrm{vN}}^{\mathrm{CJ}} \theta i_{I}\right)=\frac{D^{2}}{2}+Q+\frac{1}{\theta}\left(u_{\mathrm{vN}}^{\mathrm{CJ}} \theta \dot{l}_{I}\right) .
$$

where we use explicitly the constancy of the total enthalpy across the shock in the shock-attached frame. It follows that

$$
\left(c^{2}-\tilde{u}^{2}\right)^{(1)}=D_{C J}^{2}(\gamma-1) \Delta c-(\gamma+1) \tilde{u}^{(0)} \tilde{u}^{(1)}
$$

where

$$
\Delta c=d^{\star}+\frac{u_{\mathrm{VN}}^{\mathrm{CJ}} \theta i_{I}}{D_{\mathrm{CJ}}^{2}} .
$$

We insert this in the perturbation equation for $\tilde{u}^{(1)}$ in (2.34) and find

$$
\frac{d \tilde{u}^{(1)}}{d \ell}+\frac{\gamma}{\ell(\gamma-\ell)} \tilde{u}^{(1)}=-\frac{\gamma-1}{\ell(\gamma-\ell)} \Delta c
$$


Next we impose the sonic boundary condition at the end of the reaction zone by requiring that $\left(c^{2}-u^{2}\right)^{(0)}=\left(c^{2}-u^{2}\right)^{(1)}=0$ at $\ell=0$. For the leading order solution, which corresponds to the plane wave Chapman-Jouguet solution, this is already satisfied. At first order we obtain

$$
\left.\tilde{u}^{(1)}\right|_{\ell=0)}=-\frac{\gamma-1}{\gamma} \Delta c
$$

where we have used that $\tilde{u}^{(0)}(0)=-\gamma /(\gamma+1)$. The exact solution is

$$
\tilde{u}^{(1)} \equiv-\frac{\gamma-1}{\gamma} \Delta c=\text { const }
$$

This completes the computation of the first order main reaction layer solution.

Up to this point we have derived separate solution representations for the induction and main reaction regions and we are left with forcing these solutions to coincide in a suitable overlap region where the coordinate $\Lambda=\theta \lambda$ (from the induction zone) is large, while $\ell=\sqrt{1-\lambda}$ is close to unity. We will find that this overlap condition determines the correct value of the time derivative $i_{I}$ of the induction length.

\subsubsection{Matching and the wave speed evolution equation}

The large $-\Lambda$ - representation of the flow velocity in the shock frame derived from the induction zone solution in section 2.1.2 is

$$
\left.\frac{u}{D_{\mathrm{Cs}}}\right|_{\text {ind }}(\Lambda \rightarrow \infty)=-\frac{\gamma-1}{\gamma+1}-\frac{1}{\theta}\left\{\frac{\Lambda}{2(\gamma+1)}-\frac{2 \gamma}{\gamma^{2}-1} 2 d^{\star}+\frac{\gamma+1}{\gamma-1} d^{\star}\right\} .
$$

On the other hand, the main reaction layer solution for $\ell=\sqrt{1-\lambda}=1-1 /(2 \theta) \Lambda+\ldots$ in an expansion for $\theta \gg 1$ reads, taking into account the change of reference frames,

$$
\left.\frac{u}{D_{\mathrm{CJ}}}\right|_{\mathrm{MRL}}=\frac{\gamma-1}{\gamma+1}+\frac{1}{\theta}\left\{-\frac{\Lambda}{2(\gamma+1)}-\frac{\gamma-1}{\gamma} \Delta c+\frac{\theta i_{I}}{D_{\mathrm{CJ}}}\right\}
$$

Comparison of (2.43) and (2.44) yields the desired matching condition:

$$
\frac{2 \gamma}{\gamma^{2}-1}\left\{2 d^{\star}-\chi d^{\star}\right\}=\frac{3 \gamma-1}{\gamma(\gamma+1)} \frac{\theta i_{I}}{D_{\mathrm{cj}}}
$$

Using the explicit representation for $l_{I}\left(d^{\star}\right)$ in $(2.27)$, we find

$$
\frac{d h}{d t}=-\frac{2-\chi}{\psi(\gamma)} d^{\star}=-\frac{2-\chi}{2 \psi(\gamma)} \ln h
$$

where

$$
\psi(\gamma)=\frac{2 \gamma^{2}}{(\gamma-1)(3 \gamma-1)}
$$

This is the evolution equation announced in the introduction in (1.1) when curvature effects are absent, i.e., for $\kappa \rightarrow 0$. As discussed there, this equation describes either a collapse $(h \rightarrow 0$ in finite time) or a decomposition of the wave structure ( $h \rightarrow \infty$ as $t \rightarrow \infty$ monotonously).

Here we have described the behavior of the detonation in the near-CJ regime and we have found that the wave will leave that regime as time evolves. Hence, the evolution equation found here is not closed in the sense that the solution leaves its regime of validity. In the collapsing mode the fire accelerates continously relative to the lead shock, while keeping its CJ-deflagration 
structure. This acceleration leads to unbounded growth of the detonation speed perturbation function $d^{*}$. In the next section we consider the large amplitude continuation of this process and we will find that indeed there exists such a collapsing solution consisting of shock - induction zone fire that evolves on a persistently shrinking time scale proportional to the ever shrinking induction length. We sketch the derivation of a nonlinear second order differential equation that describes this behavior.

\section{LARGE AMPLITUDE ACCELERATIONS}

In this section we derive an asymptotic solution to the unsteady equations in $(2.1)-(2.3)$ for the reaction rate law

$$
w=B(1-\lambda) \exp \left(-\frac{E_{a}}{R T}\right)
$$

We assume that:

i) The detonation structure is acoustically decoupled from the burnt gas flow so that, at the end of the reaction zone, the flow is sonic in the frame of reference attached to the "fire", and we consider a regime, where:

ii) Deviations of the lead shock velocity from the Chapman-Jouguet speed are large, so that

$$
\frac{D-D_{\mathrm{CJ}}}{D_{\mathrm{CJ}}}=O(1)
$$

iii) time variations of the detonation speed are systematically slow measured on the instantaneous induction time scale,

$$
t_{\overline{\mathrm{I}}}=\left(\ell_{\mathrm{I}} / D\right)(\tau)
$$

where $\tau$ is a suitable, dynamically rescaled slow time variable defined by

$$
\frac{\theta \ell_{\mathrm{I}}}{D}(\tau) d \tau=d t \quad \text { with } \quad \theta(\tau)=\frac{E_{a}}{R T_{\mathrm{sh}}(\tau)}
$$

The key idea will be to find detonation solutions that are inherently unsteady and consist of a coupled shock - fire structure, which itself is decoupled from the burnt gas flow through a sonic point at the end of the fire. Now, relaxing the requirement that the mass fluxes through fire and shock are identical (as in a stationary solution) one can construct unsteady accelerating solutions in which the mass burning rate in the fire exceeds the mass flux through the shock. In this situation only part of the heat release is used up to overcome the shock dissipation. The excess chemical power is available to accelerate the detonation. Hence, we seek solutions characterized by

1) A slowly accelerating (or decelerating) lead shock,

2) an induction zone whose length depends on the instantaneous shock strength and the unsteady changes induced by the acceleration,

3) a Chapman-Jouguet deflagration that burns the gas at the end of the reaction zone.

We outline in the rest of this paper the construction of these solutions by systematic matched asymptotics for large activation energy kinetics.

Using front-attached coordinates with the dynamic time rescaling from (3.3) and a distance coordinate that scales with the current, yet unknown induction length

$$
\ell_{\mathrm{I}}(\tau) \eta=x-x_{\mathrm{sh}}(t),
$$


we are faced with the following system of balance equations:

$$
\begin{aligned}
\frac{D}{\theta} \rho_{\tau}+(u-D \dot{\mathcal{L}} \eta) \rho_{\eta}+\rho u_{\eta} & =0 \\
\frac{D}{\theta}\left(u_{\tau}+\dot{D}(\tau)\right)+(u-D \dot{\mathcal{L}} \eta) u_{\eta}+\frac{1}{\rho} p_{\eta} & =0 \\
\frac{D}{\theta}\left(E_{\tau}-\frac{p}{\rho^{2}} \rho_{\tau}\right)+(u-D \dot{\mathcal{L}} \eta)\left(E_{\eta}-\frac{p}{\rho^{2}} \rho_{\eta}\right) & =0 \\
\frac{D}{\theta} \lambda_{\tau}+(u-D \dot{\mathcal{L}} \eta) \lambda_{\eta} & =\ell_{\mathbf{I}}(\tau) w
\end{aligned}
$$

Here

$$
\begin{aligned}
& E=\frac{1}{\gamma-1} \frac{p}{\rho}+Q(1-\lambda) \\
& u=\tilde{u}-D \\
& \dot{\mathcal{L}}=\frac{1}{\theta(\tau)} \frac{\dot{\ell}_{1}}{\ell_{\mathrm{I}}}(\tau)=O(1)
\end{aligned}
$$

Before going into the details of the analysis, we outline the key idea of how to derive a detonation speed evolution equation: Having in mind the structure of a CJ-ZND detonation for large activation energy kinetics, which consists of a lead shock, an induction zone and a CJ-"fire", we seek dynamical solution with the same principal features. The lead shock velocity, however, is assumed to evolve on the instantaneous long time scale indicated in (3.3). It is followed by an induction zone, whose structure and length $\ell_{\mathrm{I}}$ remain to be determined and the structure is terminated by a CJ-deflagation that travels over the compressed post-shock state. The evolution equation for the lead shock velocity is obtained through the following steps:

1) Compute the post-shock state $\left[\rho_{0}, \tilde{u}_{0}, p_{0}\right](D(\tau))$ as a function of the instantaneous lead shock velocity $D(\tau)$, using the shock Hugoniot relations. (Recall that $\tilde{u}$ is the flow velocity in the laboratory frame!)

2) Compute the mass flux $\dot{m}_{\text {fire }}$ through a CJ-deflagration that burns this compressed post-shock gas. This mass flux is then also a function of $D$. The velocity of this fire in the laboratory frame is

$$
\dot{x}_{\text {fire }}(D)=\left(\tilde{u}_{0}+\dot{m}_{\text {fire }} / \rho_{\mathrm{sh}}\right)(D) \text {. }
$$

3) Notice that the time change of the induction length obeys

$$
\dot{\ell}_{\mathrm{I}}=D-\dot{x}_{\text {fire }}(D):=\delta \ell(D)
$$

and hence it can be expressed as a function of $D(\tau)$, too.

4) Compute, by activation energy asymptotics, the instantaneous induction length. The result, as we show in the next section, will be a relation

$$
F\left(\ell_{\mathrm{I}}, \dot{\ell}_{\mathrm{I}}, D, \dot{D}\right)=0
$$

5) Combine (B) and (C) to get first $F\left(\ell_{\mathrm{I}}, \delta \ell(D), D, \dot{D}\right)=0$, but this is effectively an equation for the induction length as a function of $D$ and $\dot{D}$ :

$$
\ell_{\mathrm{I}}=h(D, \dot{D}) \text {. }
$$

It follows, after time differentiation, that $D_{0}(\tau)$ satisfies the nonlinear second order differential equation (dropping the subscript for convenience)

$$
\frac{\partial h}{\partial \dot{D}}(D, \dot{D}) \ddot{D}+\frac{\partial h}{\partial D}(D, \dot{D}) \dot{D}=\frac{d \delta \ell}{d D}(D) \dot{D}
$$


In the next sections we provide the key ingredients of the above calculations, but an evaluation of all the formulae and an analysis and solutions of the resulting equation in (E) will be left for a future publication.

\subsection{Induction zone analysis}

Following standard procedures in large activation energy asymptotics for ignition type problems we first consider the solution in the induction region that immediately follows the leading shock front. To this end, we introduce the expansions

$$
\underline{U}=\underline{U}_{\mathrm{sh}}\left(D_{0}(\tau)\right)+\frac{1}{\theta} \underline{U}^{(1)}(\eta, \tau)+O\left(\frac{1}{\theta^{2}}\right),
$$

for the flow variables

$$
\underline{U}=(\rho, u, p, \lambda)
$$

In the expansion of the lead shock velocity,

$$
D=D_{0}(\tau)+\frac{1}{\theta^{2}} D^{(2)}+\ldots
$$

we suppress the first order term. The time evolution of the detonation velocity will be adjusted such that that a $D^{(1)}$ never occurs.

Inserting this perturbation ansatz into the governing equations in (3.5) we find that the leading order equations are satisfied automatically, while at first order the perturbations have to satisfy

$$
\begin{aligned}
\left(u_{\mathrm{sh}}-D \dot{\mathcal{L}} \eta\right) \rho_{\eta}^{(1)}+\rho_{\mathrm{sh}} u_{\eta}^{(1)} & =-D \dot{\rho}_{\mathrm{sh}}(\tau) \\
\left(u_{\mathrm{sh}}-D \dot{\mathcal{L}} \eta\right) u_{\eta}^{(1)}+\frac{1}{\rho_{\mathrm{sh}}} p_{\eta}^{(1)} & =-D\left(\dot{u}_{\mathrm{sh}}+\dot{D}\right)(\tau) \\
\left(u_{\mathrm{sh}}-D \dot{\mathcal{L}} \eta\right)\left(E_{\eta}^{(1)}-\frac{p_{\mathrm{sh}}}{\rho_{\mathrm{sh}}^{2}} \rho_{\eta}^{(1)}\right) & =-D\left(\dot{E}_{0}-\frac{p_{\mathrm{sh}}}{\rho_{\mathrm{sh}}^{2}} \dot{\rho}_{0}\right) \\
\left(u_{\mathrm{sh}}-D \dot{\mathcal{L}} \eta\right) \lambda_{\eta}^{(1)} & =\theta \ell_{\mathrm{I}}(\tau) w
\end{aligned}
$$

where we have suppressed the subscript on $D_{0}$ for simplicity of notation and where the right hand side of the last equation must, by definition of the coordinates $\eta$ and $\tau$ be of order unity (see below). Also notice that the lead shock is non-reactive, so that $\lambda_{\mathrm{sh}} \equiv 1$ and $\dot{\lambda}_{\text {sh }} \equiv 0$.

The Arrhenius reaction rate law introduced in (3.1) involves the temperature as the major determining variable. Hence it is convenient to introduce the nondimensional temperature

$$
T=\frac{p}{\rho}=T_{\mathrm{sh}}(\tau)+\frac{1}{\theta} T^{(1)}(\eta, \tau)+\ldots
$$

where obviously $T_{\mathrm{sh}} \equiv p_{\mathrm{sh}} / \rho_{\mathrm{sh}}$ and

$$
T^{(1)}=\frac{1}{\rho_{\mathrm{sh}}} p^{(1)}-\frac{p_{\mathrm{sh}}}{\rho_{\mathrm{sh}}^{2}} \rho^{(1)} .
$$

A lengthy elimination procedure based on (3.10) and (3.12) leads to the following single equation for $T^{(1)}$ :

$$
f_{1}(\eta ; \tau) T_{\eta}^{(1)}-f_{2}(\eta ; \tau)\left[\theta \ell_{1}(\tau) Q w\right]=f_{3}(\eta ; \tau)
$$


where the functions $f_{i}(\eta ; \tau)$ are given by

$$
\begin{aligned}
& f_{1}(\eta ; \tau)=\frac{1}{T_{\mathrm{sh}}(\gamma-1)}\left\{u_{*}^{2}-\gamma T_{\mathrm{sh}}\right\} \\
& f_{2}(\eta ; \tau)=\frac{1}{T_{\mathrm{sh}}}\left\{u_{*}^{2}-T_{\mathrm{sh}}\right\} \\
& f_{3}(\eta ; \tau)=D(\tau)\left[\left(u_{\mathrm{sh}}+D\right)-u_{*} \frac{\dot{\rho}_{\mathrm{sh}}}{\rho_{\mathrm{sh}}}-\frac{f_{2}}{u_{*}}\left(\dot{E}_{\mathrm{sh}}-\frac{p_{\mathrm{sh}}}{\rho_{\mathrm{sh}}^{2}} \dot{\rho}_{\mathrm{sh}}\right)\right]
\end{aligned}
$$

where

$$
u_{*}(\eta ; \tau)=u_{s h}(\tau)-(D \dot{\mathcal{L}})(\tau) \eta
$$

The square bracket in (3.13) still contains the reaction source term which is now expanded in the following fashion:

$$
\theta \ell_{\mathbf{I}} Q w=\Lambda \exp \left(\frac{T^{(1)}}{T_{\mathrm{sh}}}\right)
$$

Here

$$
\Lambda(\tau)=\theta(\tau) \ell_{\mathbf{I}}(\tau) Q B \exp (-\theta(\tau))
$$

now appears as a parameter, which must be adjusted in such a way that the usual ignition thermal runaway of $T^{(1)}$ occurs at $\eta=-1$. This is to be required according to the definition of the induction zone coordinate $\eta$ in (3.4) and will actually lead to the desired relation between $\ell_{1}, \dot{\ell}_{I}, D$ and $\dot{D}$ as announced above.

By inserting the expansion for the reaction term in (3.13) and reformulating for

$$
\Phi=\exp \left(-\frac{T^{(1)}}{T_{\mathrm{sh}}}\right),
$$

as the unknown, we find the linear first order ODE problem in $\eta$

$$
\Phi^{\prime}+g_{1}(\eta) \Phi+\Lambda g_{2}(\eta)=0, \quad \text { with } \quad \Phi(0 ; \tau)=1
$$

where

$$
\begin{aligned}
& g_{1}=\frac{f_{3}}{f_{1}}, \\
& g_{2}=\frac{f_{2}}{f_{1}},
\end{aligned}
$$

and where we have suppressed the $\tau$-dependence for simplicity of notation. The initial condition for $\Phi$ follows from the expansion of the detonation velocity $D$ in (3.9), which implies that there be no first order perturbations right behind the lead shock.

Formally, exact solutions to (3.19) can readily be written down and, given a solution family parametrized by $\Lambda$, one has to select the very value of this parameter that guarantees that $\Phi(1 ; \tau)=0$, which corresponds to a runaway of $T^{(1)}$ according to the definition of $\Phi$ in (3.18). This "eigenvalue" for $\Lambda$ will depend on $D, \dot{D}$ and $\dot{\mathcal{L}}$ via (3.14) and (3.15), the latter being a function of $\dot{\ell}_{1}, \ell_{1}$ and $D$ according to (3.6) 3 . This, combined with the definition of $\Lambda$ in (3.17), yields the relation between $\ell_{1}, \dot{\ell}_{1}, D, \dot{D}$ as announced in statement (C) above.

What is left to do is to discuss the structure of the main reaction layer and show that its representation as a CJ deflagration running over the post-shock state is appropriate to the current order of approximation. Secondly, we will have to evaluate the shock and fire Hugoniot relations in order to manifest the function $\delta \ell(D)$ statement (B). 


\subsection{The main reaction layer (fire)}

In the main reaction layer the reaction rate becomes exponentially large in comparison with its magnitude in the induction zone. It is thus even more justified to seek a steady-state - or quasisteady - solution in a suitable reference frame. The onset of the rapid heat release occurs in an extremely narrow layer near the end of the induction zone, where $\eta=-1$. The time scale for changes of the fire structure is dictated here by the slow time scaling introduced in (3.3), so that after introduction of a suitable rescaled space coordinate

$$
\xi=\frac{1}{H(\theta)}(\eta+1)
$$

with $H(\theta)$ being exponentially large in $\theta$, the leading order solution in the fire is determined by the one-dimensional plane wave structure equations

$$
\begin{aligned}
\left(\hat{u}^{(0)} \rho^{(0)}\right)_{\xi} & =0 \\
\hat{u}^{(0)} \hat{u}_{\xi}^{(0)}+\frac{1}{\rho^{(0)}} p^{(0)}{ }_{\xi} & =0 \\
E_{\xi}^{(0)}-\frac{p^{(0)}}{\left(\rho^{(0)}\right)^{2}} \rho_{\xi} & =0 \\
\hat{u}^{(0)} \lambda_{\xi}^{(0)} & =\ell_{\mathbf{I}}(\tau) w .
\end{aligned}
$$

Hence, the leading order solution does correspond to a plane steady deflagration in the "fire"frame, characterized by $\eta \equiv-1$. The solution is uniquely determined be (i) the pre-deflagration pressure, temperature and fuel composition and (ii) by an additional constraint on the mass flux through the fire. Here we assume that this deflagration sees sonic outllow of the burnt gases in its own reference frame, so that it is a CJ-deflagration and guarantees that the shock - induction zone - fire structure decouples from the background flow.

Hence, as pointed out above, in order to describe the influence of the fire on the solution, we need to formulate in detail the $\mathrm{CJ}$-deflagration Hugoniot-conditions to find the mass burning rate as a function of the post-shock state, or equivalently, as a function of the lead shock velocity.

\subsection{Shock- and fire Hugoniot relations}

The shock Hugoniot conditions can be extracted form any standard text book on gasdynamics. We recount them here, nevertheless, because the non-dimensionalisation introduced in section 2 is not usually used in text books and thus we prepare the jump relations for use in the present notation.

Let primes denote dimensional quantities and consider the dimensionless shock density and shock pressure

$$
\begin{aligned}
& \rho^{*}=\frac{\rho_{\mathrm{sh}}^{\prime}}{\rho_{0}^{\prime}}, \\
& p^{*}=\frac{p_{\mathrm{sh}}^{\prime}}{p_{0}^{\prime}} .
\end{aligned}
$$

Furthermore let

$$
M^{2}=\frac{D^{2}}{c_{0}^{2}} \quad \text { with } \quad c_{0}^{2}=\gamma \frac{p_{0}}{\rho_{0}},
$$

be the shock Mach number. Then the standard text-book Hugoniot jump relations read

$$
\begin{aligned}
\rho^{*} & =\frac{(\gamma+1) M^{2}}{2+(\gamma-1) M^{2}} . \\
p^{*} & =\frac{2 \gamma M^{2}}{\gamma+1}-\frac{\gamma-1}{\gamma+1},
\end{aligned}
$$


These relations are re-written in the present non-dimensionalization as follows: First we have the scaled pre-shock pressure

$$
\hat{p}_{0}=\frac{p_{0}^{\prime}}{\rho_{0}^{\prime} D_{C J}^{\prime 2}}
$$

and then

$$
\begin{aligned}
& \rho_{\mathrm{sh}}=\frac{(\gamma+1)}{(\gamma-1)+2 \gamma \hat{p}_{0} / D^{2}}, \\
& p_{\mathrm{sh}}=D^{2}\left(\frac{2}{\gamma+1}-\frac{\gamma-1}{\gamma+1} \frac{\hat{p}_{0}}{D^{2}}\right), \\
& u_{\mathrm{sh}}=-\frac{D}{\rho_{\mathrm{sh}}} .
\end{aligned}
$$

The post-shock velocity in the front-attached frame of reference has been derived by using the conservation of mass. This finishes the formulation of the shock Hugoniot conditions.

Now we consider the CJ-deflagration running over the state given by the shock conditions in (3.27). The mass flux through this flame, or rather the relative velocity between the fire and the mass elements in the post-shock state, can be expressed by a straight-forward, but very tedious calculation in the following fashion:

$$
u_{\mathrm{defl}}:=\frac{\dot{m}_{\mathrm{deff}}}{\rho_{\mathrm{sh}}}=\frac{\gamma-1+2 \gamma \pi}{(\gamma+1)(1-\gamma \pi)} \sqrt{2\left(\gamma^{2}-1\right) Q}
$$

where

$$
\pi=\frac{2 \gamma+\left(4 \gamma-(\gamma-1)^{2}\right) q^{*}-(\gamma+1) \sqrt{q^{*}\left(4 \gamma+(\gamma+1)^{2} q^{*}\right)}}{2\left(\gamma^{2}+2 \gamma(\gamma-1) q^{*}\right)}
$$

with

$$
q^{*}=2 \frac{\gamma-1}{\gamma+1} \frac{Q}{p_{\mathrm{sh}} / \rho_{\mathrm{sh}}} .
$$

The relations in (3.28) - (3.30) yield readily the desired fire velocity in the laboratory frame as expressed in statement $(A)$ above as a function of $\rho_{\mathrm{sh}}(D), p_{\mathrm{sh}}(D)$ and the heat release parameter Q. In fact,

$$
\dot{x}_{\text {fire }}=D+u_{\mathrm{sh}}(D)+u_{\text {defl }}\left(\rho_{\mathrm{sh}}(D), p_{\mathrm{sh}}(D), Q\right) .
$$

or

$$
\dot{\ell}_{\mathrm{I}}(D)=D-\dot{x}_{\mathrm{fire}}=-\left(u_{\mathrm{sh}}(D)+u_{\mathrm{den}}\left(\rho_{\mathrm{sh}}(D), p_{\mathrm{sh}}(D) ; Q\right)\right) .
$$

This finishes the collection of formulae that are the ingredients of the second order nonlinear detonation dynamics equation as discussed at the beginning of this section.

Detailed evaluations of these formulas, suitable limit considerations for the near-CJ limit, for the passage through CJ conditions and for turning points in the evolution of $D(\tau)$, i.e., the analysis of acceleration - deceleration transitions and vice versa, will follow in a forthcoming publication. Here we have achieved our goal of outlining the construction of a detonation dynamics equation that relies on no mre and no less than a large activation energy limit analysis and the assumption of slow evolution on the instantaneous ignition delay time scale as expressed in (3.3).

\section{SUMMARY AND CONCLUSION}

In this paper we propose a large amplitude theory for the dynamics of detonation waves based on large activation energy asymptotics. Assuming the detonation structure to evolve on a timescale that is systematically by one order larger, in terms of the activation energy, than the instantaneous induction time, we arrive at a nonlinear second order evolution equation for the detonation shock velocity $D$ :

$$
\frac{\partial h}{\partial \dot{D}}(D, \dot{D}) \ddot{D}+\frac{\partial h}{\partial D}(D, \dot{D}) \dot{D}=\frac{d \delta \ell}{d D}(D) \dot{D}
$$


Here $h(D, \dot{D})$ is essentially the instantaneous induction length and a dot indicates a derivative with respect to the scaled time variable $\tau$ defined by the differential relation

$$
\frac{\theta \ell_{1}}{D}(\tau) d \tau=d t \quad \text { with } \quad \theta(\tau)=\frac{E_{a}}{R T_{\mathrm{sh}}(\tau)}
$$

Here $E_{a}, T_{\text {sh }}, R, \ell_{1}$ are the activation energy of the Arrhenius reaction rate law considered, the instantaneous post-shock temperature, the instantaneous induction length and the gas constant, respectively. We have outlined here the key ideas in deriving this equation. Detailed analyses of the function $h(D, \dot{D})$, which is the result of an involved induction zone analysis, and of the associated features of the equation in (4.1) will be reserved for future work.

We are aware that the assumption of a slow time evolution is a severe one, considering the chaotic instability of large activation energy detonations. Nevertheless, we believe that the present considerations should be taken seriously, because of the following fact: Any fast time stability theory based on a steady state solution might be erroneous, because the true background long time behavior may follow the slow-time dynamics described here. It would be interesting to study fast time perturbations that evolve on top of the large amplitude dynamical modes obtained here.

Also, we emphasize that in the present theory the "fire" moves relative to the lead shock of the detonation with a speed comparable to the detonation speed itself! Hence, the time of collapse is comparable to the passage time of a particle through the induction zone. We conclude that the present dynamical modes, due to the strong nonlinearity of the induction chemistry, override any evolution that would rely on a single time scale based on the induction length for a specific detonation strength. In fact, the evolution described here involves a highly nonlinear dynamical time rescaling so that the true characteristic time of evolution shrinks rapidly as the wave accelerates.

\section{REFERENCES}

[1] Abuseif G.E., Toong T.Y., "Theory of Unstable Onedimensional Detonations", Comb. \& Flame 45 (1982) 67-94

[2] Bourlioux A., Majda A.J., "Theoretical and numerical structure of twodimensional detonations", Comb. E Flame 90 (1992) 211-229

[3] He L., Clavin P., "Stability and Nonlinear Dynamics of One-Dimensional Overdriven Detonations in Gases", submitted to J. Fluid Mech. January (1995)

[4] Klein R., "Curved Detonations in Explosive Gas Mixtures with High Temperature Sensitivity ", Intl. Conf. on Combustion, 80th Birthday Memorial for Ya.B. Zeld'dovic, Moskau, June 1994

[5] Yao J., Stewart D.S., "On the normal shock velocity-curvature relationship for materials with large activation energy", Comb. E Flame (1994) to appear

[6] Klein R., Krok , Shepherd J.E., "Investigation of Curved Quasisteady Detonations Using Asymptotic Analysis and Detailed Chemical Kinetics", to be submitted (1995)

[7] Buckmaster J., "Pressure Transients and the Genesis of Transverse Shocks in Unstable Detonations", Comb. Sci. \& Technol. 61 (1988) 1-20

[8] Klein R., Stewart D.S., "The relation between curvature, rate state dependence and detonation velocity", SIAM J.Appl.Math. 53 (1993) 1401-1435 